\title{
Sources of color-word interference in the Stroop color-naming task
}

\author{
ROBERT W. PROCTOR \\ Auburn University, Auburn, Alabama 36830
}

\begin{abstract}
The results of several previous experiments have suggested that interference in the Stroop color-naming task is greater for color words that are also members of the set of possible color-naming responses. However, all previous experiments had strength of association to the concept of color and, in most cases, word frequency confounded with membership in the response set. The present experiments controlled for these factors and obtained strong evidence that interference is substantially greater when the printed words are also members of the response set. In addition, it was shown that for color words that are not from the response set the amount of interference is a function of the strength of association of the words with the concept of color. It was concluded that there is selective activation of the colornaming response set in memory. Internal activation of the remaining color terms is a function of how strongly they are associated with other color-naming responses.
\end{abstract}

In the Stroop color-naming task, subjects are shown colored forms that spell incongruent color names. The time to name the color of the form is longer than for a control condition in which the forms are rectangles or a series of asterisks or Xs. In recent years, many variations of the Stroop task have been used to examined mental processes (see Dyer, 1973, for a review).

Klein (1964) and Scheibe, Shaver, and Carrier (1967) conducted two similar studies in which they varied the relationship of several types of verbal forms to the color names (see also Dalrymple-Alford, 1972; Fox, Shor, \& Steinman, 1971; Hochman, 1967). In addition to the standard Stroop-task condition in which the color words came from the same set of colors as those being named (referred to from this point as the same-set condition), the conditions examined included ones in which the forms spelled color words from a different set of colors than those being named (the different-set condition), words that implicated specific colors, words with no specific color meaning, and nonsense syllables. The widely cited finding of both studies was that all conditions produced interference in color naming (as compared to a control condition), with the amount of interference a direct function of the semantic or associative relatedness of the words to color names.

One curious outcome of the Klein and Scheibe et al. experiments is that color words from the color-

This research was supported in part by Auburn University Grant-in-Aid 76-151. I would like to thank Camille Powell for assistance in data collection. Requests for reprints should be sent to Robert W. Proctor, Department of Psychology, Auburn University, Auburn, Alabama 36830. naming response set produced approximately twice the interference of color words from a different set. In fact, the difference in the amount of interference produced by these two conditions was greater than the difference between the different-set color words and nonsense syllables or uncommon words with no color implications. If the basis of the interference is the degree of association to color names (or to the concept of color), the interference of the different-set words would be expected to be of approximately the same magnitude as the interference of the same-set color words.

One possible reason for the difference in interference between the same-set and different-set color words is that, in both the Klein and Scheibe et al. experiments, the different-set color words were also of lower average frequency and less strongly associated with other color-naming responses and the concept of color. Fox, Shor, and Steinman (1971) controlled for average frequency (but not strength of association) and still found a large, though somewhat smaller, difference between the same-set and different-set conditions. While the differences in average frequency and strength of association to color responses most likely contributed to the large difference in interference produced by same-set and different-set color words in the Klein and Scheibe et al. experiments, it is unlikely that these factors accounted for the whole difference.

It appears, then, that a large component of the interference observed in the standard Stroop colornaming task is specific to members of the response set. Recent evidence indicates that prior activation of the internal unit corresponding to the printed word results in an increased amount of interference 
(Warren, 1972, 1974). Thus, greater interference from members of the response set would be expected if there is selective activation of the set of possible color-naming responses.

Although the Klein and Scheibe et al. experiments suggest an effect of response set, they do not provide critical evidence in this regard, since in both experiments the same-set and different-set conditions also differed in their strength of association with the concept of color, as well as in average frequency. Critical evidence regarding the effect, if any, of membership in the response set can be obtained by comparing two conditions that differ only in whether the color words come from the response set.

Two experiments in the literature approximate the critical test of response-set interference in the Stroop task, yet both have crucial procedural differences that make them inadequate in this regard. Harrison and Boese (1976) had subjects respond with words related to the ink color in which the stimulus was printed, rather than responding with the color name. For example, if the color of the form was red, the response would be "blood." They compared time to respond with the color-related names when the printed words were from the response set vs. when they were from a different set of color-related words. Interference was greater when the printed words came from the response set. There is a problem, however, in extending the results obtained by Harrison and Boese to the standard color-naming situation, since the related-response task they employed very likely involves an additional stage (retrieval of the appropriate response from memory once the color has been named) at which the response-set interference might occur.

Morton (1969) also found evidence for responseset interference with an analogue of the Stroop task. Specifically, the task required sorting cards into categories on the basis of the number of symbols on the card. Morton found that when the symbols were copies of a number that was different from the number of symbols on the card, there was interference with the sorting task. More interference was produced when the symbols were numbers from the sorting response set than when they were other numbers.

The primary problem with Morton's experiment regarding the question of response-set interference in the Stroop task is that at least one result obtained by Morton suggests that the numerosity task differs in some fundamental respect from the Stroop task. Specifically, Morton found that auditorially presented digits interfere with the sorting task, whereas existing evidence indicates that there is no interference of auditorially presented color words with color naming (e.g., Thackray \& Jones, 1971). In addition, it appears that the numbers from the response set (response set: $1,2,3,4,5$, and 6 ; only 3,4 , and 5 were used as printed numbers) were more closely associated with the alternative numerosity responses than were the numbers that were not members of the response set $(7,8$, and 9$)$.

In summary, while the existing evidence strongly suggests that membership of the printed word in the response set is an important factor influencing the amount of interference in the Stroop color-naming task, there is no conclusive evidence in this regard. Experiment 1 was designed to obtain the appropriate evidence by comparing the amount of interference produced in same-set and different-set conditions similar to those examined by Klein and Scheibe et al., with the exception that the color-word sets were equated as closely as possible for average frequency of occurrence in the English language, strength of association with the concept color, and strength of association within and between sets.

\section{EXPERIMENT 1}

\section{Method}

Subjects and Design. Twenty-four upper-division undergraduates served as subjects in fulfillment of a class requirement. All subjects participated in each of the three experimental conditions, making the design a single factor within-subjects design.

Stimulus materials. Lists of 80 stimulus items were employed. Each list was mounted on three columns (one column of 26 stimuli and the other two of 27 stimuli) on a single sheet of white paper. The stimuli on any page could be printed in one of four ink colors. Half of the subjects received lists in which the ink colors were red, yellow, purple, and brown; the other half of the subjects received lists in which the ink colors were orange, green, blue, and black. The color sets were selected so that they were composed of familiar color names that occur approximately equally often, on the average, in the English language (Kučera \& Francis, 1967). In addition, the sets were chosen so that they were approximately equally strongly associated with the concept of color (Battig \& Montague, 1969) and so that the associations between color names were approximately equally strong within and between sets (Jenkins, 1970). ${ }^{1}$

For each ink-color set, lists of stimuli for three conditions were constructed. The conditions differed in the relationship of the printed forms to the ink-color set (also referred to as the colornaming response set). For the control condition, each form was a series of four Xs. The remaining two conditions were similar in that the forms spelled color words that were incongruent with the ink color in which the stimulus was printed. The conditions differed in that for the same-set condition the spelled words were also members of the color-naming response set, whereas for the different-set condition the spelled words came from the set of color names that were not being employed as the response set. An example of a same-set stimulus would be the word "yellow" printed in red ink, while a different-set stimulus might be the word "blue" printed in red ink.

Lists were constructed in the following manner. First, three random orders of 80 ink colors were prepared. The only constraints on order were that a color could not be the same as for the preceding stimulus and that each color occurred equally often in each half of a list. For the control lists, the three lists of ink colors were printed in forms of four Xs. For the sameset condition, each ink color was randomly paired with an incongruent color word. The restrictions were that the spelled word could not be the same as the previous word and that each word was 
paired equally often with each ink color. The different-set lists were constructed by substituting the color words for the same-set condition with the color words that were not members of the response set according to the following pairings: orange-red, greenyellow, blue-purple, black-brown. Thus, a stimulus that was the word "yellow" printed in red ink in the same-set list would be changed to the word "green" printed in red ink for the differentset list. All subjects received different list orders of ink colors in each condition, with ink-color lists counterbalanced across subjects.

Procedure. Subjects were instructed to proceed through the list as fast as possible without making errors. They were told to start with the left-hand column and, when finished with it, to proceed successively through the remaining columns. Each subject received lists from only one of the two ink-color sets. Half of the subjects received lists from one of the ink-color sets; half received lists from the other. As commonly practiced in Stroop-task studies, all subjects were presented the control condition first. The two experimental conditions were counterbalanced for order so that half of the subjects received the same-set condition next, followed by the different-set condition, while half received the opposite order. All subjects were told in advance which condition would be presented on the trial. They were then handed the appropriate sheet face down. On signal from the experimenter, they turned it over and proceeded to name the ink colors as fast as possible. The experimenter measured the time required to complete the list with a stopwatch.

\section{Results}

The time it took each subject to complete the list in each condition was recorded. The mean response times (in seconds) are shown in Table 1.

Two planned comparisons were employed to analyze the response-time data. The first analysis compared the control condition to the two colorword conditions to determine whether the Stroop effect had occurred. This comparison was highly significant, $F(1,46)=236, p<.001$, indicating that mean response time was faster in the control condition than in the two experimental conditions.

The second comparison was between the same-set and the different-set conditions. This comparison was also significant, $F(1,46)=22.9, \mathrm{p}<.001$, indicating that it took longer for subjects to name the list when the color words were from the same response set as the color names than when they were not. Thus, interference is greater when the color words and the color-naming responses both come from the same set.

\section{EXPERIMENT 2}

The results of Experiment 1 clearly indicate that there is less interference in naming a list of ink colors when the spelled color words come from a different set than the color-naming responses. When the continuous list procedure is employed with a small set of stimuli, as in Experiment 1, the same-set condition includes many instances in which the color word for a particular stimulus is the same as the ink color of the previous stimulus, and vice versa. Since sequential relationships appear to interfere with re-
Table 1

Mean Response Times in Seconds for Experiment 1

\begin{tabular}{ccc}
\hline Control & Same Set & Different Set \\
\hline 44.9 & 74.1 & 65.2 \\
\hline
\end{tabular}

sponding (e.g., Dalrymple-Alford \& Budayr, 1966; Neill, 1977), it is possible that the existence of these relationships in the same-set list but not in the differentset list could account for the entire difference in interference between these two conditions.

Many recent studies of the Stroop task have employed a procedure in which reaction times (RTs) to name single stimuli are obtained, rather than the time to name an entire list. The single-stimulus procedure eliminates the problem of having to suppress and then say, or vice versa, a particular response in succession. In addition, since the single-stimulus procedure allows random intermixing of stimulus conditions, the stimulus sequence can be constructed so that neither the ink color nor the printed word for each trial are used as either the printed word or the ink color on the immediately preceding and succeeding trials. Thus, the single stimulus procedure should minimize any sequential effects from one stimulus to the next. One purpose of Experiment 2 was replication of the results of Experiment 1 with the single stimulus procedure to ensure that the results of Experiment 1 could not be attributed solely to the sequential relationships present in the same-set condition.

Although a large difference in interference between the same-set and different-set conditions was obtained in Experiment 1, it was apparently of a smaller magnitude than the differences obtained by Klein and Scheibe et al. when the different-set color words were also of lower frequency and less strongly associated with the concept of color than the sameset words. Thus, the next logical step is to determine more directly whether strength of association or frequency has any influence on the amount of interference color words produce once the effect of response set has been taken into account. The second purpose of Experiment 2 was to examine this question by including a different-set condition composed of uncommon color words that are neither as strongly associated with the concept of color nor of as high frequency as the common color words employed in Experiment 1. Uncommon color words were chosen for a number of reasons, with the primary reason being that they should provide the strongest test of whether the factors of frequency and strength of association to color have any effect. ${ }^{2}$

\section{Method}

Subjects and Design. Twenty-five undergraduate students enrolled in psychology courses served as subjects. All subjects re- 
ceived credit toward their course grades for participation. Data from one subject was discarded due to his refusal to refrain from humming and making other extraneous noises that interfered with the recording of vocal RT. The design was a single-factor, withinsubjects design.

Apparatus and Stimuli. Stimuli were presented in a two-channel tachistoscope (Gerbrands, Model T-AB). Warning interval and stimulus durations were timed by the tachistoscope's control unit. The subject's naming response activated a voice-operated relay (Grason-Stadler, Model E7300A-1). RTs were recorded to the nearest millisecond on a digital clock-counter (Lafayette, Model 54519-A), which measured the time from presentation of the stimulus to activation of the voice-operated relay.

The stimuli were forms printed in one of four ink colors. The stimuli were mounted individually on index cards. They were centered on the cards, subtending a visual angle of approximately $50^{\prime}$ vertically and $1^{\circ} 57^{\prime}-3^{\circ} 15^{\prime}$ horizontally. As in Experiment 1 , half of the subjects received stimuli for which the colors were red, yellow, purple, or brown, while the other half received stimuli that were orange, green, blue, or black. Stimulus forms came from the three different conditions examined in Experiment 1 [(1) Xs; (2) incongruent color words from the same set as the ink colors; (3) common color words from the set that was different from the ink colors], and a fourth condition of uncommon color words from a different set than the ink colors. The uncommon color words were scarlet, amber, lavender, and tan (employed as the uncommon color words for the orange, green, blue, and black color list); and navy, emerald, salmon, and ebony (employed as the uncommon different-set color words for the list with ink colors of red, yellow, purple, and brown). The uncommon color words are not as strongly associated with the concept of color as the common color words (Battig \& Montague, 1969), nor do they have as strong interassociations with the common color names as the common color names do with one another (Jenkins, 1970). Average frequency of occurrence in the English language is also less for the uncommon color words than for the common color words (Kučera \& Francis, 1967).

Orders of trials were constructed for both color sets. In this experiment, stimuli from the different conditions were randomly intermixed. The method of construction will be described for one set (red, yellow, purple, brown), followed by a description of how the order for the second color set was derived from the first. Two random orders of 48 trials ( 12 for each condition) were constructed. The primary restrictions on ordering were that each condition occurred equally often in each half of the list and that neither the ink color nor the printed word on a trial was the same as either the ink color or the word on the previous trial (although there were a few immediate repetitions of the control pattern, since repetition of conditions was allowed). In addition, all colors occurred three times in each condition. For the same-set condition, each color was paired once with each of the three color words corresponding to the other members of the response set. For the different-set/common condition, each color was paired with three of the common color words that were not members of the response set (orange, green, blue, and black). The particular pairings of colors with words was determined in the same manner as in Experiment 1. For the different-set/uncommon condition, each color was paired with the three members of the uncommon word set that corresponded to those members from the different-set/ common condition with which the colors were paired (salmonorange, emerald-green, navy-blue, ebony-black). For example, the word "blue" would be printed in red once in the different-set/ common condition, while the word "navy" would be printed in red once in the different-set/uncommon condition. Subjects received a total of 96 trials (both list orders), with the order of the two lists of trials counterbalanced across subjects. The orders for the second color set (orange, green, blue, black) were constructed by substituting the colors as in Experiment 1 and by changing the words for the same-set condition and the different- set/common condition in the same manner. The different-set/ uncommon words for the second ink-color set were scarlet, amber, lavender, and tan. These were substituted in the stimulus orders for salmon, emerald, navy, and ebony, respectively. Thus, the same basic orders of trials were used for the second set of colors, although the particular color-word combinations were changed.

Procedure. The subjects were instructed that their task was to name, as fast as possible, the ink color in which stimulus forms were printed. They were told the four possible ink colors that might occur, and that the forms would either spell another color name or would be a series of Xs. Furthermore, they were told that the spelled color names would come from a set including the ink colors and the eight other color names (the differentcommon and different-uncommon sets). The instructions stressed that the subjects should not employ methods to blur the pattern (such as squinting), and that they should refrain from making extraneous noises that might activate the voice-operated relay.

The subjects were instructed to fixate in the center of the lighted blank field of the tachistoscope. Each trial began with a "ready" signal from the experimenter. Simultaneous with this signal, the experimenter closed a switch that initiated the trial sequence. Five hundred milliseconds later a colored form was presented, centered in the viewing field, for a 1-sec duration. Following the subject's response, the experimenter recorded the RT and changed the stimulus in preparation for the next trial. Approximately $15 \mathrm{sec}$ elapsed between successive trials. The occasional trials (less than 1\%) on which either the response did not stop the timer or an extraneous noise stopped the timer were rerun later in the session.

At the start of the session, the subjects were given 10 practice trials naming color-word stimuli. The sequence of 96 experimental trials was then presented.

\section{Results and Discussion}

The error rate for the same-set condition was $1.6 \%$. Only one subject made an error in the differentset/common condition, while no errors were made for the other two conditions. Median RTs were obtained for each subject as a function of stimulus condition for the correct responses. The means of these data are shown in Table 2.

Specific planned comparisons were performed as in Experiment 1. The comparison of the control condition to the other three conditions was highly significant, $F(1,69)=241.8, p<.001$, indicating that the color words did interfere with color naming. The second comparison compared the same-set condition to the different-set/common and different-set/ uncommon conditions. This comparison was also significant, $F(1,69)=36.2$, $p<.001$, indicating that the words from the same response set caused more interference than the color words that were not from

Table 2

Mean Reaction Times in Milliseconds for Experiments 2 and 3

\begin{tabular}{ccccc}
\hline & & Same & \multicolumn{2}{c}{ Different Set } \\
\cline { 3 - 5 } $\begin{array}{c}\text { Experi- } \\
\text { ment }\end{array}$ & Control & Set & Common* & Uncommon $\dagger$ \\
\hline 2 & 614.5 & 750.1 & 721.1 & 690.9 \\
3 & 687.1 & 795.5 & 771.8 & 747.2 \\
\hline
\end{tabular}

*High association

tLow association 
the response set. The last comparison was between the different-set/common condition and the differentset/uncommon condition. Again, the comparison was significant, $F(1,69)=13.6, p<.001$, indicating that the uncommon color words produced less interference with color naming than the common color words.

The results of Experiment 2 that were obtained with the single-stimulus procedure clearly replicate those of Experiment 1 obtained with the whole list procedure. That is, more interference was produced when the color words were also members of the color-naming response set. Since this outcome was obtained with the single-stimulus procedure, it is clear that something other than sequential relationships between color words and ink colors on successive trials must account for the greater interference observed when the color words are members of the response set. It appears that the response-set interference reflects selective activation of the colornaming response set in memory.

The second major outcome of Experiment 2 is that even when the effect of response set is taken into account, common color words still produce more interference than uncommon color words. Since common color words differ from uncommon ones in both frequency of usage and strength of association with the concept of color, either or both of these factors may have contributed to the difference in interference. Evidence exists that both word frequency and association strength influence the amount of interference produced by noncolor words (e.g., Klein, 1964), suggesting that both of these factors contributed to the difference between common and uncommon color words. However, while it seems reasonable to assume that the effect of frequency is relatively independent of whether or not the printed words come from the category of color names, it is less reasonable to assume that the pattern of interference due to the associational relationship is similar for both members and nonmembers of the category of color names. Thus, Experiment 3 was designed to provide more direct evidence regarding the influence of strength of association to the concept of color when the printed words spell color names.

\section{EXPERIMENT 3}

In Experiment 3, an attempt was made to control for frequency of occurrence to rule out the possibility that the difference in interference between common and uncommon color words observed in Experiment 2 could be attributed solely to nonassociative processes. The conditions were basically the same as those of Experiment 2, with the exception that the color words in the two different-set conditions were all high-frequency color words. However, the different-set conditions still differed in strength of association with the concept of color.

\section{Method}

Apparatus and stimuli. The apparatus was the same as in Experiment 2, and stimuli were prepared to the same specifications. However, only one set of ink colors (yellow, pink, orange, and purple) was employed. Since the primary purpose of Experiment 3 was to compare iwo different-set conditions composed of common color words that differed in strength of association with the concept of color, the different-set words were selected first, and then the particular ink colors used were selected from the remaining basic colors (Berlin \& Kay, 1969).

The four conditions examined (control, same-set, different-set/ high-association, different-set/low-association), were the same as those of Experiment 2, with the exception that the two differentset conditions were composed of common color words of approximately equal frequency in the language (Kučera \& Francis, 1967) that differed in the strength of association with the concept of color (Battig \& Montague, 1969) and the degree with which they were associated to members of the ink-color set (Jenkins, 1970). The color words for the different-set/high-association condition were red, blue, and green; while the words for the different-set/ low-association condition were black, white, and gray. Since only three words were used in each different-set condition, each word was paired once with each ink color (rather than with only three ink colors, as in Experiments 1 and 2).

Subjects and Procedure. Twenty students enrolled in undergraduate psychology courses served as subjects. All subjects received credit toward their course grades for participation. The procedure was identical to that of Experiment 2.

\section{Results and Discussion.}

The error rate was $2.5 \%$ for the same-set condition and approximately $0.5 \%$ for each of the other conditions. Median RTs were obtained for each subject as in Experiment 2, and the means of these data are shown in Table 2.

The same planned comparisons were performed as in Experiment 2. The comparison of the control condition to the other three conditions was significant, $F(1,79)=101, p<.001$, as was the comparison of the same-set condition to the two different-set conditions, $F(1,79)=16.4, p<.001$. The latter comparison indicates that, as in Experiments 1 and 2, color words from the response set caused more interference than other color words. The most important comparison was that of the different-set/highassociation condition to the different-set/lowassociation condition. This comparison was also significant, $F(1,79)=5.71, p<.025$, indicating that even when equated for frequency of occurrence, color words more strongly associated with color produce more interference.

In addition to being less strongly associated with the concept of color than the high-association color words, the low-association color words in Experiment 3 were achromatic terms. This confounding of chromatic-achromatic with association strength was necessary to control for word frequency. However, as a consequence, it might be argued that the dif- 
ference in interference occurred solely because the achromatic terms cannot properly be regarded as members of the color category. Evidence that little interference occurs in an achromatic version of the Stroop task (both colors and words come from the set black, white, and gray; Dyer, 1971; Smith \& Borg, 1964) might be interpreted as support for this argument. However, at least three lines of evidence indicate that achromatic colors are classified with other color terms. First, subjects commonly include these terms when generating color names (Battig \& Montague, 1969). Second, Dyer (1971) has obtained evidence indicating that the relative lack of interference in the achromatic task is apparently due to the ability to name achromatic shades faster than colors, not to any difference in the way achromatic color words are processed. Third, when black was a member of the response set in Experiment 2, the printed word "black" produced an amount of interference that was approximately equal to that produced by other members of the response set. Thus, the evidence suggests that the achromatic-chromatic distinction does not in itself account for the difference in interference produced by the high- and lowassociation color-word sets.

\section{CONCLUSIONS}

One of the primary outcomes of the reported experiments is that color words that are also members of the color-naming response set produce more interference than color words that are not members of the response set. While several previous studies have strongly suggested that membership in the response set is an important determinant of interference in the Stroop task, the present experiments are the first to clearly demonstrate it.

The present results regarding membership in the response set corresond closely to Morton's (1969) findings with the numerosity-sorting task that were discussed earlier. As such, the general explanation developed by Morton appears to apply to the present situation. That explanation is that the effect of response set reflects activation of the internal recognition units corresponding to the possible color-naming responses. The increased interference occurs for printed words that correspond to the activated response-set members because the response to the printed word is elicited more quickly. Morton has proposed that, as a consequence, the response to the printed word is entered into a serial response buffer prior to the correct color-naming response more often than when the words are not from the activated response set. An alternative possibility is that the interference occurs over ambiguity about which one of the two activated responses to enter into the response buffer (Seymour, 1977). Regardless of the specific locus of the interference, it appears that the difference in interference between color words that are from the response set and ones that are not is attributable to prior activation of the color-naming response set.

Posner and Snyder (1975a, 1975b) have proposed that there are two qualitatively different activation processes that can facilitate processing of signals. One of the activation processes is an automatic process that occurs whenever a familiar item, such as a word, is presented. This activation process does not require conscious attention. The second type of activation does not occur automatically, it occurs only when a person consciously attends to signals. Either of these two activation processes could produce the response-set effect observed in the present experiments.

Warren $(1972,1974)$ has shown that automatic activation has a strong influence on response times in the Stroop task. He found that auditory presentation of a word immediately preceding a Stroop trial on which the printed word is the same as or related to the preceding word results in increased interference. The automatic activation (and, as a consequence, the increased interference) appears to dissipate within approximately $30 \mathrm{sec}$ (Warren, 1972).

It is obvious that automatic activation may contribute at least in part to the response-set effect when the whole-list procedure is employed (Experiment 1). With this procedure, subjects process successive stimuli within $1 \mathrm{sec}$ of each other. Automatic activation would be expected to be greater for the list in which the printed words are also members of the colornaming response set, since both the words and the ink colors activate the same set of recognition units, while only the printed words activate the relevant recognition units when the words are not from the color-naming response set.

On the other hand, it does not appear that automatic activation can account for the effect of response set with the single stimulus procedure (Experiments 2 and 3 ). In the experiments employing this procedure, the printed word on each trial had not occurred as either a word or ink color on the previous trial. Since approximately $15 \mathrm{sec}$ intervened between each trial, at least $30 \mathrm{sec}$ had elapsed since the previous occurrence of a particular color name. Thus, if automatic activation dissipates within $30 \mathrm{sec}$, as Warren's (1972) data suggest, it would not have been a factor in Experiments 2 and 3 . It is possible that a long-term automatic activation occurred due to the multiple presentations of each color stimulus. However, the interpretation that appears most reasonable is that the activation observed with the single stimulus procedure reflects conscious attention to the response set. This interpretation coincides with the fact that subjects appear to rehearse the response set, and often report anticipating particular colors on some trials. 
The previous studies that manipulated membership in the response set confounded this factor with strength of association to the concept of color and other color-naming responses. Thus, the second major problem addressed by the present experiments was whether the strength with which color words are associated with the concept of color influences the amount of interference when the words are not members of the response set. Two alternative patterns of activation, corresponding to the effect or lack of effect of association strength, seemed possible. First, all members of the color category (at least the common ones) might receive an equal amount of secondary activation. That is, through their links with the concept of color, the response-set members might secondarily activate the entire structure of the concept. Second, the degree to which the remaining color terms would be activated might depend on the strength of association to the concept of color or the activated response-set members. The evidence clearly supports the latter alternative in that color words that are less strongly associated with the concept of color produce less interference than more common instances of the category. This outcome is similar to results obtained with noncolor words (e.g., Klein, 1964), suggesting that the structural relation of color terms to one another is similar to that of color terms to noncolor concepts.

There are two possible explanations of the association-strength results. First, it is possible that the activation pattern is similar to a "typicality" effect (e.g., Rips, Shoben, \& Smith, 1973). That is, it is possible that the pattern of activation does, in fact, reflect the strength of association to the color concept, with more typical members receiving more activation. A second possible explanation is that the pattern of activation reflects the strength with which the color names are associated with the activated members of the response set. The present experiments do not distinguish between these two alternatives. However, Warren's (1974) results showing the associational nature of the priming of noncolor words suggests that the latter alternative is most likely.

\section{REFERENCES}

Battig, W. F., \& Montague, W. E. Category norms for verbal items in fifty-six categories: A replication and extension of the Connecticut Category Norms. Jourmal of Experimental Psychology Monographs, 1969, 80, No. 3, Part 2, 1-46.

BERLIN, B., \& KAY, P, Basic color terms: Their universality and evolution. Berkeley and Los Angeles: University of California Press, 1969.

Dalrymple-Alforn, E. C. Associative facilitation and interference in the Stroop color-word task. Perception \& Psychophysics, 1972, 11, 274-276.

Dalrymple-Alford, E. C., \& BUdayr, B. Examination of some aspects of the Stroop color-word test. Perceptual and Motor Skills, 1966, 23, 1211-1214.

DYER, F. N. A comparison of chromatic and achromatic versions of the Stroop color-word test. Psychonomic Science, 1971, 22, 235-237.

Dyer, F. N. The Stroop phenomenon and its use in the study of perceptual, cognitive, and response processes. Memory \& Cognition, 1973, 1, 106-120.

Fox, L. A., Shor, R. E., \& Steinman, R. J. Semantic gradients and interference in naming color, spatial direction, and numerosity. Journal of Experimental Psychology, 1971, 91, 59-65.

HARrison, N. S., \& Boese, E. The locus of semantic interference in the "Stroop" color-naming task. Perception \& Psychophysics, 1976, 20, 408-412.

Hochman, S. H. The effects of stress on Stroop color-word performance. Psychonomic Science, 1967, 9, 475-476.

Jenkins, J. J. The 1952 Minnesota word association norms. In L. Postman \& G. Keppel (Eds.), Norms of word association. New York: Academic Press, 1970.

KLEIN, G. S. Semantic power measured through the interference of words with color-naming. American Journal of Psychology, 1964, 77, 576-588.

KUČERA, H., \& Francis, W. W. Computational analysis of present-day American English. Providence, R.I: Brown University Press, 1967.

Morton, J. Categories of interference: Verbal mediation and conflict in card sorting. British Journal of Psychology, 1969, 60. 329-346.

NEILL, W. T. Inhibitory and facilitory processes in selective attention. Journal of Experimental Psychology: Human Perception and Performance, 1977, 3, 444-450.

Posner, M. I., \& SNYder, C. R. R. Facilitation and inhibition in the processing of signals. In P. M. A. Rabbitt \& S. Dornic (Eds.), Attention and performance V. New York: Academic Press, 1975. (a)

Posner, M. I., \& Snyder, C. R. R. Attention and cognitive control. In R. L. Solso (Ed.), Information processing and cognition: The Loyola Symposium. Hillsdale, N.J: Erlbaum, 1975. (b)

Rips, L. J., Shoben, E. J., \& Smrth, E. E. Semantic distance and the verification of semantic relations. Journal of Verbal Learning and Verbal Behavior, 1973, 12, 1.20.

Scheibe, K. E., Shaver, P. R., \& Carrier, S. C. Color association values and response interference on variants of the Stroop test. Acta Psychologica, 1967, 26, 286-295.

SEYmour, P. H. K. Conceptual encoding and locus of the Stroop effect. Quarterly Journal of Experimental Psychology, 1977, 29, 245-265.

SmITH, B. J. W., \& Borg, G. W. V. The problem of retesting in the serial colour-word test. Psychological Research Bulletin, IV: 6 (Lund University, Sweden), 1964.

ThackRay, R. I., \& Jones, K. N. Level of arousal during Stroop performance: Effects of speed, stress, and "distraction." Psychonomic Science, 1971, 23, 133-135.

WARREN, R. E. Stimulus encoding and memory. Journal of Experimental Psychology, 1972, 94, 90-100.

W ARREN, R. E. Association, directionality, and stimulus encoding. Journal of Experimental Psychology, 1974, 102, 151-158.

\section{NOTES}

1. The color words for which association norms are provided in the Jenkins (1970) norms are red, yellow, blue, green, black, and white. The responses to these words were used as the primary basis for the associational manipulations in all experiments.

2. The terminology "strength of association to the concept of color" is employed for Experiments 2 and 3 with the knowledge that the critical factor may more specifically be strength of association to the set of possible color-naming responses (see the Conclusions section for a more detailed consideration of this issue).

(Received for publication December 19, 1977; revision accepted February 22, 1978.) 